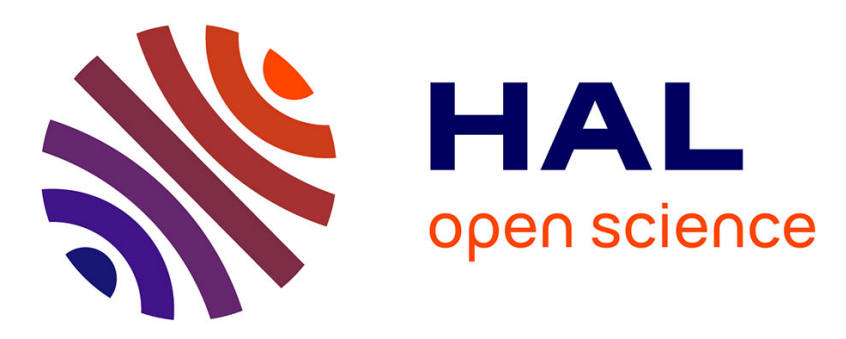

\title{
A comparative Evaluation of Length Estimators of digital Curves
}

David Coeurjolly, Reihnard Klette

\section{To cite this version:}

David Coeurjolly, Reihnard Klette. A comparative Evaluation of Length Estimators of digital Curves. IEEE Transactions on Pattern Analysis and Machine Intelligence, 2004, 26 (2), pp.252-258. 10.1109/TPAMI.2004.1262194 . hal-00185088

\section{HAL Id: hal-00185088 https://hal.science/hal-00185088}

Submitted on 6 Nov 2007

HAL is a multi-disciplinary open access archive for the deposit and dissemination of scientific research documents, whether they are published or not. The documents may come from teaching and research institutions in France or abroad, or from public or private research centers.
L'archive ouverte pluridisciplinaire HAL, est destinée au dépôt et à la diffusion de documents scientifiques de niveau recherche, publiés ou non, émanant des établissements d'enseignement et de recherche français ou étrangers, des laboratoires publics ou privés. 


\section{A Comparative Evaluation of Length Estimators of Digital Curves}

\author{
David Coeurjolly and Reinhard Klette
}

\begin{abstract}
This paper compares previously published length estimators in image analysis having digitized curves as input. The evaluation uses multigrid convergence (theoretical results and measured speed of convergence) and further measures as criteria. This paper also suggests a new gradient-based method for length estimation, and combines a previously proposed length estimator for straight segments with a polygonalization method.
\end{abstract}

Index Terms—Length estimator, digital geometry, curve length, multigrid convergence.

\section{INTRODUCTION}

THE digitization of curves or boundaries has been studied in image analysis for about 40 years [1]. Since these first studies, many algorithms have been proposed to estimate the length of a digitized curve. Some approaches are based on local metrics, such as the weighted metrics, other approaches are based on polygonalizations of digital curves, e.g., directed on subsequent calculations of maximum-length digital straight segments (DSSs) or of minimum length polygons (MLPs). This paper compares previously published length estimators by defining and applying different performance measures for a sample set of 2D curves. We also propose and include a new length estimator based on calculated gradients.

The computational problem of estimating the length of a digital curve based on maximum-length DSS segmentation is as follows: the input is a sequence of chain codes $i(0), i(1), \ldots$ with $i(k) \in A=\{0, \ldots, 7\}, k \geq 0$. An offline algorithm takes finite words $u \in A^{\star}$ as input, and performs the required calculations, e.g., to decide whether $u$ is a DSS or not. An online algorithm reads successive chain codes $i(0), i(1), \ldots$ and always provides a result up to the most recent input value $i(n)$, e.g., it decides whether $i(0), i(1), \ldots, i(n)$ is still a DSS, and if not, then it initializes a new DSS with $i(n-1) i(n)$. After completing one maximum-length DSS, its length estimate (typically, the Euclidean distance between its end vertices) is added to the length estimator of the curve. An offline algorithm is linear iff it runs in $\mathcal{O}(n)$ time, i.e., it performs at most $\mathcal{O}(|u|)$ basic computation steps for any input word $u \in A^{\star}$. An online algorithm is linear iff it uses on the average a constant number of operations for any incoming chain code symbol.

The classification into online and offline algorithms may also be applied to other procedures for length estimation. An obvious benefit of local metrics based approaches is that they support linear online implementations. This method has frequently been suggested in the image analysis literature for length estimation, combined with proposals of local weights to improve these estimations. However, it is known that these methods are only of limited use if multigrid convergence is applied as a selection criteria.

DSS-based polygonalization is a popular method in image analysis, allowing us to transform digital boundaries into polygonal objects (see Fig. 1a). Linear offline algorithms for DSS recognition

- D. Coeurjolly is with the Laboratoire LIRIS, Université Lumière Lyon 2, 5 Av. Pierre Mendès France, 69676 Bron CEDEX, France.

E-mail: david.coeurjolly@univ-lyon2.fr.

- R. Kletteis with CITR, The University of Auckland, Tamaki campus, Building 731, Morrin Road, Glen Innes, Auckland 1005, New Zealand.

E-mail:r.klette@auckland.ac.nz.

Manuscript received 20 Mar. 2002; revised 13 Sept. 2002; accepted 18 Dec. 2002.

Recommended for acceptance by E. Hancock.

For information on obtaining reprints of this article, please send e-mail to: tpami@computer.org, and reference IEEECS Log Number 116125.

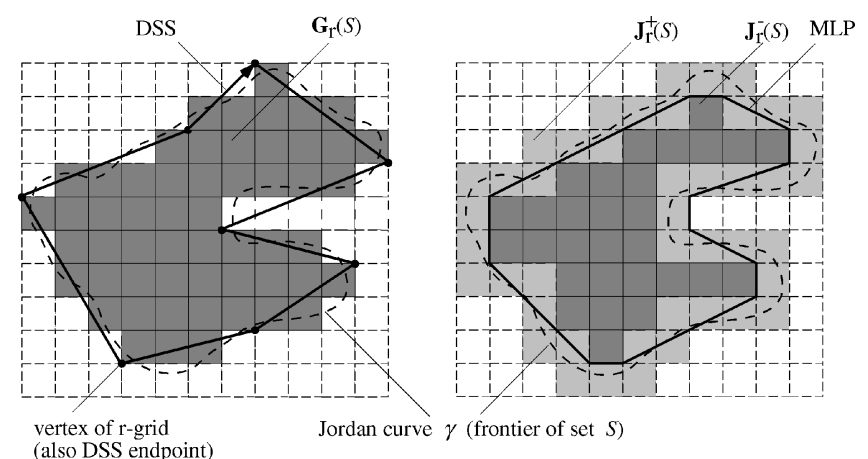

(a)

(b)

Fig. 1. (a) Segmentation of a 4-path into a sequence of maximum-length DSSs. (b) MLP-approximation between two polygonal boundaries.

were published in 1981 in [5] and in 1982 in [8]. A linear offline algorithm for cellular straight segment recognition, based on convex hull construction, is briefly sketched in [7]. Two linear online algorithms for DSS recognition were published in 1982 in [6]; one of them is an online version of the offline algorithm published in [5].

The general problem of decomposing a digital curve into a sequence of DSSs, which includes DSS recognition as a subproblem, is discussed in, e.g., [15], [16], [20], [27]. Obviously, linear online DSS recognition algorithms will support linear decomposition algorithms, but the application of a linear offline algorithm for inputsequences of increasing length, i.e., first for $i(0)$, then for $i(0) i(1)$, then for $i(0) i(1) i(2)$, etc., leads to quadratic runtime behavior.

MLP-based polygonalization provides a third method, which is not yet of widespread use in image analysis. An MLP-approximation [22], [28] calculates a minimum-length polygon circumscribing a given (closed) inner boundary (given by a sequence of chain codes) and being in the interior of an outer boundary (typically, in Hausdorff-Chebyshev distance 1 to the inner boundary). This polygon is also known as relative convex hull [3] and calculations of relative convex hulls have a history in computational geometry and robotics (see Fig. 1b).

As a fourth method, we also introduce gradient-based length estimation in this paper that may be seen as an extension of the DSS-based approach. The notions online, offline, or linear time apply for algorithms following any of these four design strategies.

In this paper, we present a comparative evaluation of length estimators covering these four types of strategies. We are especially interested in evaluating these algorithms (and underlying methodologies) with respect to the accuracy of length estimation. Multigrid convergence is one option of characterizing this accuracy, and experimental studies provide another way for performance testing. Our experiments are directed on illustrating accuracy and stability of the chosen algorithms on convex and nonconvex curves (see Fig. 2 for the used test data which had been proposed in [27]). These given Jordan curves of known length are digitized for increases in grid resolution, allowing to study and illustrate experimental multigrid convergence.

In our experiments, we digitize these curves in grids varying between $30 \times 30$ and $1,000 \times 1,000$. For the digitization of a planar Jordan curve (up to a given grid resolution), we may adopt any of the digitization models known in the image analysis literature.

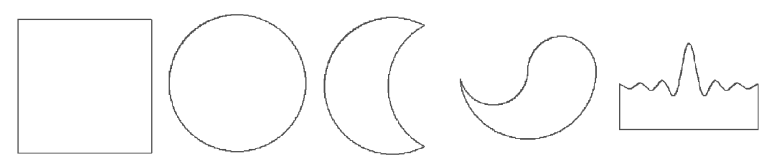

Fig. 2. Test data set. 

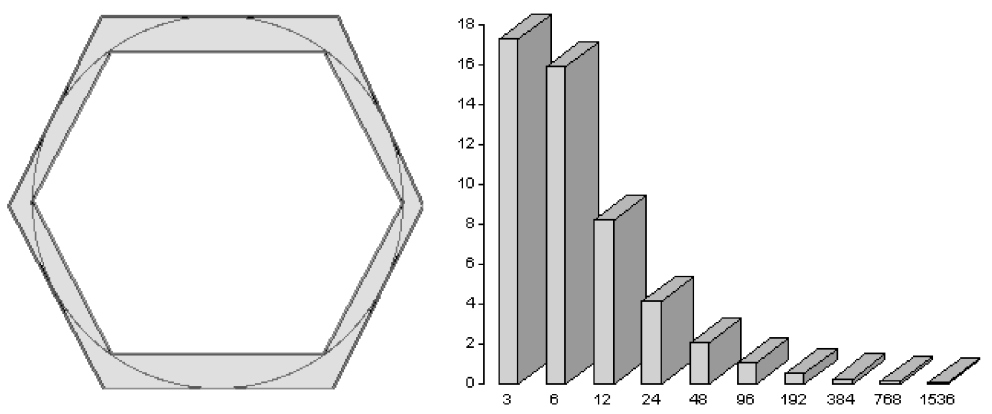

Fig. 3. Left: Inner and outer hexagon approximating a circle. Right: percentage errors for perimeter estimation using inner $n$-gons.

We assume an orthogonal grid with grid constant $0<\theta \leq 1$ in the Euclidean plane $\mathbb{R}^{2}$, i.e., $\theta$ is the uniform spacing between grid points parallel to one of the coordinate axes. Let $r=1 / \theta$ be the grid resolution and the r-grid $\mathbb{Z}_{r}^{2}$ has resolution $r$, defined by r-points whose coordinates are $(\theta \cdot i, \theta \cdot j)$, with $i, j \in \mathbb{Z}$. Now, we consider a Jordan curve $\gamma:[0,1] \rightarrow \mathbb{R}^{2}$, being the topological frontier of a set $S$ in the Euclidean plane. Let $D_{r}(\gamma)$ be an $r$-digitization of $\gamma$ in $\mathbb{Z}_{r}^{2}$ (see Fig. 1):

Definition 1. In this paper, an r-digitization of curve $\gamma$ is one of the following:

1. the cyclic 4-path $\sigma_{r, 4}(\gamma)$ or 8-path $\sigma_{r, 8}(\gamma)$ of r-grid points following an r-grid-intersection digitization of $\gamma$, see [2] for the original definition of grid-intersection digitization with $r=1$;

2. the cyclic 4- or 8-path following vertices of r-grid squares in the frontier of the Gauss digitization $\mathbf{G}_{r}(S)$ of set $S$ in the $r$-grid, where $\mathbf{G}_{r}(S)$ is the union of all $r$-grid squares having their centroids in the given set $S$, or

3. the closed difference set between outer and inner Jordan digitization $\mathbf{J}_{r}^{+}(S)$ and $\mathbf{J}_{r}^{-}(S)$, i.e., $\operatorname{cl}\left(\mathbf{J}_{r}^{+}(S) \backslash \mathbf{J}_{r}^{-}(S)\right.$ ), where $\mathbf{J}_{r}^{+}(S)$ is the union of all $r$-grid squares having a nonempty intersection with the given set $S$ and $\mathbf{J}_{r}^{-}(S)$ is the union of all $r$-grid squares contained in the topological interior of the given set $S$.

See, e.g., [30] for more details and historic citations for these digitization methods. In the following, let $\mathcal{L}(\gamma)$ be the length of the curve $\gamma$. We denote by $\mathcal{E}$ an estimated property. Assume that $\mathcal{E}$ is defined for digitizations $D_{r}(\gamma)$, for $r>0$ and all curves $\gamma$ in a class $\Gamma$ of curves. In this paper, we only consider the class $\Gamma$ of all Jordan curves in the Euclidean plane.

Definition 2. The estimated property $\mathcal{E}$ is said to be multigrid convergent toward $\mathcal{L}$ with respect to digitization model $D_{r}$ and class $\Gamma$ iff for any curve $\gamma \in \Gamma$ we have that $\mathcal{E}\left(D_{r}(\gamma)\right)$ converges to $\mathcal{L}(\gamma)$, for $r \rightarrow \infty$. More formally: $\left|\mathcal{E}\left(D_{r}(\gamma)\right)-\mathcal{L}(\gamma)\right| \leq \kappa(r)$ with $\lim _{r \rightarrow \infty} \kappa(r)=0$. The order $O(1 / \kappa(r))$ denotes the speed of this convergence.

Multigrid convergency of estimated properties is a standard constraint in numerical mathematics for discrete versions of "continuous" properties. We conclude this introduction with a citation of a historic example in [34]:

Example 1. In ancient mathematics, Archimedes and Liu Hui [10] estimated the length $\mathcal{L}(\gamma)$ of a circular curve $\gamma$. Liu Hui used regular $n$-gon approximations, with $n=3,6,12,24,48,96, \ldots$, see left-hand side of Fig. 3. In case of $n=6$, it follows $3 \cdot d<$ $\mathcal{L}(\gamma)<3.46 \cdot d$ for diameter $d$, and for $n=96$ it follows that $223 / 71<\pi<220 / 70$, i.e., $\pi \approx 3.14$. The used method is mathematically correct because the perimeters of inner and outer regular $n$-gons converge toward the circle's perimeter for $n \rightarrow \infty$. For example, assume radius $\rho$ and let $n_{m}=3 \times 2^{m}$ with $m \geq 0$ and consider the inner $n_{m}$-gon $P_{n_{m}}$, having $n_{m}$ edges of length $p_{m}$. The length of these edges satisfies the recursion equation

$$
p_{m+1}=\rho \sqrt{2 \rho^{2}-\rho \sqrt{4 \rho^{2}-p_{m}^{2}}}
$$

It follows that the perimeter of $P_{n_{m}}$ equals $n_{m} \cdot p_{m}$ and $\kappa\left(n_{m}\right) \approx \frac{2 \pi \rho}{n_{m}}$, for $m \geq 1$ The function $\kappa(n)$ defines the speed of convergence, which is linear in this case. Altogether, the estimated length converges toward the true length with respect to regular $n$-gon approximation and the class of all circular curves.

\section{Local Metrics}

Local metrics were historically the first attempts toward a solution of the length estimation problem in image analysis. These algorithms apply to digital curves defined by options 1 or 2 in Definition 1 and can be viewed as shortest path calculations in weighted adjacency graphs of pixel locations. Weights have been designed with the intention of approximating the Euclidean distance. For example, horizontal and vertical moves in the orthogonal $r$-grid may be weighted by $1 / r$ and diagonal moves may be weighted by $\sqrt{2} / r$. More generally, a chamfer metrics definition first lists elementary moves and then associates weights to each move, see [12], [13], [18].

In order to make length estimation as accurate as possible, the use of statistical analysis has been suggested to find those weights that minimize the mean square error between estimated and true length of a straight segment. For example, [13] presents a best linear unbiased estimator (BLUE for short) for straight lines and defines the following length estimator (CM from "chessboard metric" since that is the name of the local metric it is based on):

$$
\mathcal{E}_{C M}\left(\sigma_{r, 8}(\gamma)\right)=\frac{1}{r} \cdot\left(0.948 \cdot n_{i}+1.343 \cdot n_{d}\right),
$$

where $n_{i}$ is the number of isothetic steps and $n_{d}$ of diagonal steps in the $r$-grid. We include this estimator into our comparative study. It fails to be multigrid convergent for length estimations of digitized arcs or curves (see Section 6).

We also implemented the cornercount length estimator [9] (CC for short) and include this estimator into our comparisons:

$$
\mathcal{E}_{C C}\left(\sigma_{r, 8}(\gamma)\right)=\frac{1}{r} \cdot\left(0.980 \cdot n_{i}+1.406 \cdot n_{d}-0.091 \cdot n_{c}\right),
$$

where also another type of elementary steps is counted: $n_{c}$ is the number of odd-even chaincode transitions in a digital arc or curve and the weights have been optimized (again as a best linear unbiased estimator).

\section{Polygonal DSS-APPROACHES}

Many algorithms have been published for the DSS recognition problem. Digital curves are again defined by options 1 or 2 in Definition 1. DSS-approaches are based on characterizations of digital lines, such as syntactic chain code properties [5], [8], arithmetical properties defining tangential lines [20], properties of 


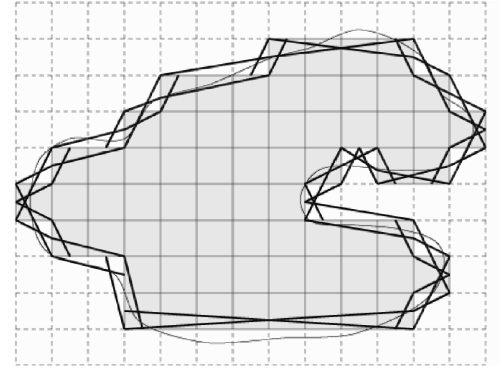

(a)

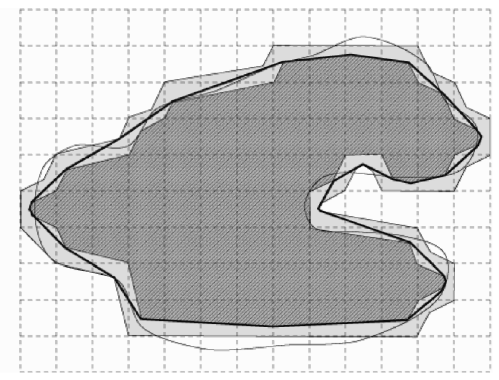

(b)

Fig. 4. In [31], (a) construction of approximating sausage and (b) resulting AS-MLP curve.

feasible regions in the (dual) parameter space [14], [16], or use linear programming tools such as the Fourier-Motzkin algorithm [21]. All these algorithms present a solution for deciding whether a given sequence of $r$-grid points is a DSS or even for segmenting a digital curve into a sequence of maximum-length DSSs. The length estimator $\mathcal{E}_{D S S}$ is then defined by the length of the obtained polyline. Note that DSS-approximations are not uniquely defined, they vary from method to method and depend, in general, upon the chosen start point and the orientation of curve tracing.

In our comparative study, we include two representative implementations of DSS-based length estimators: If the digital curve is defined as an 8-curve, we use the Debled-Reveillès algorithm [20] and call it the $\mathcal{E}_{D R-D S S}$ estimator. In our implementation, we strictly follow the algorithm as described in [20]. If the digital curve is defined as a 4-curve, we consider a length estimator based on Kovalevsky's algorithm [15] and call it the $\mathcal{E}_{V K-D S S}$ estimator. We use the algorithm as implemented for, and detailed, in [27].

These two DSS-based length estimators are known to be multigrid-convergent for convex Jordan curves $\gamma$ [19], [30], [32]. Given a simply-connected compact set $S$ in the Euclidean plane and a grid resolution $r$, the $r$-frontier $\partial \mathbf{G}_{r}(S)$ of $S$ is uniquely determined (i.e., the frontier of the Gauss digitization $\mathbf{G}_{r}(S)$ with respect to the topology of the Euclidean plane). Note that an $r$-frontier may consists of several nonconnected curves even in the case of a bounded convex set $S$. A set $S$ is r-compact iff there is a number $r_{0}>0$ such that $\partial \mathbf{G}_{r}(S)$ is just one (connected) curve, for any $r \geq r_{0}$.

Theorem 1 [30]. Let $S$ be a convex, r-compact polygonal set in $\Re^{2}$.

Then, there exists a grid resolution $r_{0}$ such that for all $r \geq r_{0}$, any DSS approximation of the $r$-frontier $\partial \mathbf{G}_{r}(S)$ is a connected polygon with perimeter $p_{r}$ satisfying the inequality

$$
\left|\mathcal{L}(\partial(S))-p_{r}\right| \leq \frac{2 \pi}{r}\left(\varepsilon_{D S S}(r)+\frac{1}{\sqrt{2}}\right)
$$

This theorem and its proof can be found in [30]. The proof is based to a large extent on material given in [19]. The value of $r_{0}$ depends on the given set and $\varepsilon_{D S S}(r) \geq 0$ is an algorithm-dependent approximation threshold specifying the maximum HausdorffChebyshev distance (generalizing the Euclidean distance between points to a distance between sets of points) between the $r$-frontier $\partial \mathbf{G}_{r}(S)$ and the constructed (not uniquely specified-see comments above) DSS approximation polygon. Assuming $\varepsilon_{D S S}(r)=1 / r$, it follows from (4) that the upper error bound for DSS approximations is characterized by ${ }^{1}$

$$
\frac{2 \pi}{r^{2}}+\frac{2 \pi}{r \cdot \sqrt{2}} \approx \frac{4.5}{r} \quad \text { if } \quad r \gg 1 \quad \text { (i.e., } r \text { is large). }
$$

1. Let $\kappa(r)=2 \pi / r^{2}+2 \pi / r \cdot \sqrt{2}$. Then, it follows that $\kappa(r) \rightarrow \pi \sqrt{2}$ as $r \rightarrow \infty$.
Grid resolution $1 / r$ is assumed in the chord property defined in [2], where a DSS is assumed to be a finite 8-path. In the case of using cell complexes, it is appropriate to consider a finite 4-path as a DSS iff its main diagonal width is less than $\sqrt{2}$, see [11], [17], [19].

Dorst and Smeulders [16] specified a most probable original (MPO, for short) estimation method with superlinear convergence $O\left(r^{-1.5}\right)$ of asymptotic length estimation for the case of digitized straight lines. Let $n$ be the length of a digital straight segment and $p / q$ the best possible rational estimate of its slope. Then,

$$
\left.\mathcal{E}_{M P O}\left(\sigma_{r, 8}(\gamma)\right)\right)=\frac{1}{r} \cdot\left(n \sqrt{1+(p / q)^{2}}\right) .
$$

Of course, it can also be applied, in addition, to a maximum-length DSS segmentation method, e.g., defining a DSS-based length estimator DR-DSS-MPO: apply Debled-Reveillès algorithm for DSS segmentation followed by summing all MPO-length-estimates of all DSSs.

\section{Polygonal MLP-Approaches}

MLP-based length estimators consider a situation where a given simple digital curve $C$ is described by two discrete curves $\gamma_{1}$ and $\gamma_{2}$, bounding sets $S_{1}$ and $S_{2}$, respectively, such that $S_{2}$ is contained in the interior $S_{1}^{\circ}$ of set $S_{1}$ and $C$ is contained in $B=S_{1} \backslash S_{2}^{\circ}$. Digital curves are defined, in this case, by option 3 in Definition 1. $S_{1}$ and $S_{2}$ are given as $\mathbf{J}_{r}^{+}(S)$ and $\mathbf{J}_{r}^{-}(S)$. The task consists of calculating the MLP which is contained in $B$ and circumscribes $\gamma_{2}$. The length estimator $\mathcal{E}_{M L P}$ is then defined by the length of this (uniquely defined [22], [25]) MLP.

In our comparative study, we include two representative implementations of MLP-based length estimators: the gridcontinua MLP approach of [22], [25] has been derived for the model of using inner and outer Jordan digitization, and defining $B$ to be the difference set between outer and inner Jordan digitization. We use the MLP algorithm as reported in [27]. We call it the $\mathcal{E}_{S Z-M L P}$ estimator.

As another MLP-method, we include the approximation-sausage $M L P$ approach of [28], [31] defining the $\mathcal{E}_{A S-M L P}$ estimator which actually involves a parameter $\delta$, with $0<\delta \leq .5 / r$. This parameter specifies a polygon $A_{r}^{\delta}(S)$ which is referred to as the approximating sausage of the $r$-frontier (see Fig. 4 a for $\delta=1 / 2$ ). The width of such an approximating sausage depends on the value of $\delta$. An AS-MLP curve for approximating the boundary of $S$ is defined as being a shortest closed curve $\gamma_{r}^{\delta}(S)$ lying entirely in the interior of the approximating sausage $A_{r}^{\delta}(S)$ and encircling the internal boundary of $A_{r}^{\delta}(S)$ (see Fig. 4b). It follows that such an AS-MLP curve $\gamma_{r}^{\delta}(S)$ is uniquely defined and that it is a polygonal curve defined by finitely many straight segments. We have taken $\delta=.5 / r$ for the experimental evaluation.

Both cited MLP-based length estimators are known to be multigrid-convergent for convex Jordan curves $\gamma$ [22], [28], [31]. 


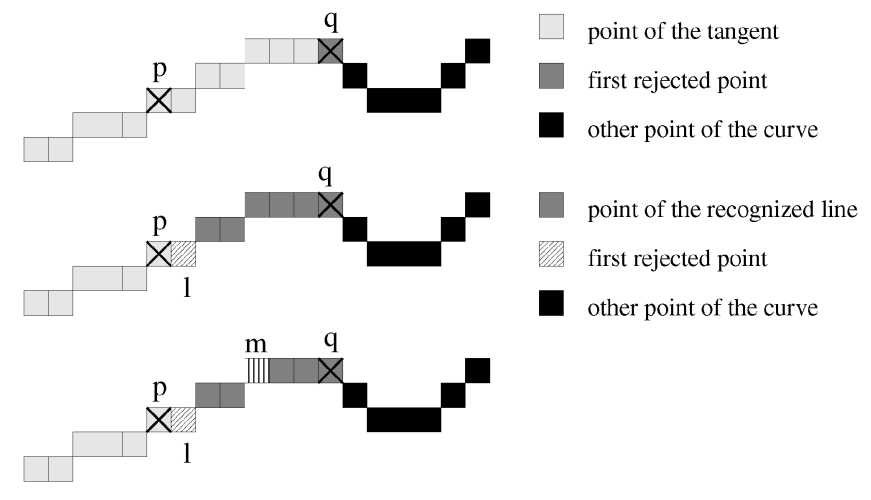

Fig. 5. Illustration of optimal-time computation of a tangent of a discrete curve.

Theorem 2 [28]. Let $S$ be a bounded, r-compact convex polygonal set. Then, there exists a grid resolution $r_{0}$ such that for all $r \geq r_{0}$ it holds that any AS-MLP approximation of the $r$-frontier $\partial \mathbf{G}_{r}(S)$, with $0<\delta \leq .5 / r$, is a connected polygon with a perimeter $l_{r}$ and

$$
\left|\mathcal{L}(\partial S)-l_{r}\right| \leq(4 \sqrt{2}+8 * 0.0234) / r=5.844 / r .
$$

For the case of SZ-MLP approximations, there are several convergence theorems in [25], showing that the perimeter of the SZ-MLP approximation is a convergent estimator of the perimeter of a bounded, convex, smooth, or polygonal set in the Euclidean plane. The following theorem is basically a quotation from [25]; it specifies the asymptotic constant for SZ-MLP perimeter estimates. A sequence of $r$-squares, where any $r$-square has exactly two edge-neighboring $r$-squares in the sequence, is called a one-dimensional grid continuum.

Theorem 3 [25]. Let $\gamma$ be a (closed) convex curve in the Euclidean plane which is contained in a one-dimensional grid continuum of r-squares, for $r \geq 1$. Then, the SZ-MLP approximation of this one-dimensional grid continuum is a connected polygonal curve with length $l_{r}$ satisfying the inequality:

$$
l_{r} \leq \mathcal{L}(\gamma)<l_{r}+\frac{8}{r} .
$$

Theorems 1, 2, and 3 show that the DSS error bound of $4.5 / r$ is smaller than the AS-MLP bound $5.844 / r$ and the AS-MLP is smaller than the SZ-MLP bound $8 / r$.

\section{Normal Vector-Based Approach}

We use a normal vector integration process to estimate the length of a curve. Let $\vec{n}:[0,1] \rightarrow \mathbb{R}^{2}$ denote the normal vector field associated with curve $\gamma$. The length of $\gamma$ can be expressed as

$$
l(\gamma)=\int_{0}^{1} \vec{n}(s) d s .
$$

The main idea of the normal vector-based approach consists of using discrete estimates of products $\vec{n}(s) d s$. This method was originally proposed by Ellis et al. in [4]. We present here both a linear time algorithm and a multigrid convergence proof of such an estimator.

The discrete tangent on a digital curve was proposed in [23], and it is based on a chosen DSS definition (see Fig. 6): The discrete tangent at point $p$ of a digital curve is the longest DSS centered at point $p$. Note that this definition is applicable whatever DSS definition (and related recognition algorithm) is chosen. A straightforward application of a linear online DSS recognition algorithm (at any point $p$ ) leads to an $O\left(n^{2}\right)$ solution. However, the optimization proposed in [26] allows to compute all tangents in linear time, assuming a cellular approach (i.e., a grid square is a 2 -cell which has four 0 -cells as its vertices and four 1-cells as its edges) for defining pixel locations.
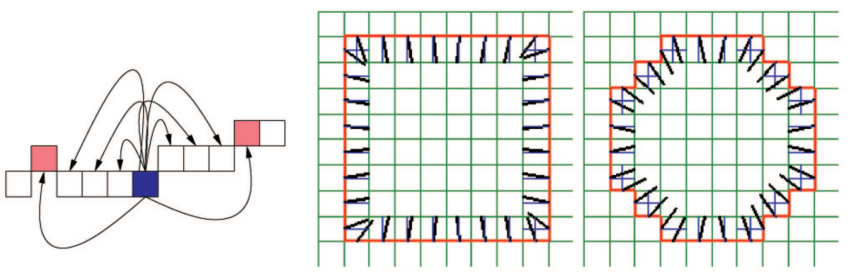

Fig. 6. Illustration of the discrete tangent calculus and examples of discrete normal vector computations for a square and a circle: black (bold) vectors are estimated normals and blue (thin) vectors are elementary normals.

The basic idea of this optimal algorithm is illustrated in Fig. 5: Discrete tangent parameters at a point $p$ of a discrete curve will not change for some "neighboring" pixels. More precisely, we consider the discrete tangent at point $p$ and the first rejected point is denoted by $q$ (i.e., the first point that does not belong to the DSS, see Fig. 5, top). Then, we compute a DSS from $q$ to $p$ and define point $l$ to be the first rejected point during this process (Fig. 5, middle). Finally, Feschet and Tougne [26] prove that for all pixels between $p$ and the middle $m$ of segment $[q l]$ (Fig. 5, bottom), the discrete tangent parameters do not change. They finally propose an efficient algorithm whose time complexity is linear in the number of pixels that computes the discrete tangent at each point of the discrete curve.

This Feschet-Tougne algorithm computes a discrete tangent at each 0 -cell of a given curve (an alternating sequence of 0 and 1-cells). Thus, we can use this for calculating the discrete normal vector at each 0-cell as a unit vector perpendicular to the discrete tangent.

We define the normal vector $\tilde{n}$ associated to a 1-cell as the mean vector of both vectors calculated at its two neighboring 0-cells. We also define an elementary normal vector $n_{e l}$ to a 1-cell as the unit vector perpendicular to this 1-cell (see Fig. 6). Hence, the discrete version of (9) is:

$$
\mathcal{E}_{T A N}\left(D_{r}(\gamma)\right)=\sum_{s \in \mathcal{S}} \tilde{n}(s) \cdot n_{e l}(s),
$$

where "." denotes the scalar product and $\mathcal{S}$ is the set of all 1-cells of $D_{r}(\gamma)$, which is assumed to be an alternating sequence of 0 -cells and 1-cells. The main idea of this approach is to compute the contribution of each 1-cell to the global length estimation by projecting the 1-cell according to the direction of the normal. In [33], it is shown that length estimator $\mathcal{E}_{T A N}$ is multigrid convergent for convex Jordan curves.

Theorem 4 [33]. Let $\gamma$ be a $C^{2}$ curve with bounded curvature, then both the discrete normal vector direction and the gradient-based length estimator are multigrid convergent.

The speed of multigrid convergence and the maximum error bound of the gradient-based length estimator require further studies to be determined.

\section{Evaluation}

We compare the chosen length estimator algorithms based on practical experiments and available theoretical results. Regarding algorithmic complexity note that all are linear online algorithms. From all the possible MOP-extensions of DSS and MLP methods, we only report on DR-DSS-MOP in this section. Theoretical studies should answer the following questions:

- multigrid convergence: Is the estimator multigrid convergent for convex curves (if "yes," we are also interested in an analysis of convergence speed)?

- discrete: Does the core of the algorithm only deal with integers?

- unique: Does the result not depend on initialization (such as start point, orientation of scan, etc.)? 
TABLE 1

Length Estimators Used in This Comparison

\begin{tabular}{|c|c|c|c|c|c|}
\hline Method & multigrid & discrete & unique & 3D extension & References \\
\hline DR-DSS & yes & yes & no & {$[32]$} & {$[20]$} \\
\hline DR-DSS-MPO & yes & yes & no & - & {$[20]+[16]$} \\
\hline VK-DSS & yes & yes & no & - & {$[15]$} \\
\hline SZ-MLP & yes & yes & yes & {$[29]$} & {$[3],[22]$} \\
\hline AS-MLP & yes & no & no & - & {$[28],[31]$} \\
\hline TAN & yes & yes & yes & - & {$[4]$, this article } \\
\hline Local metrics & no & depends & yes & {$[24]$} & {$[12],[13]$} \\
\hline
\end{tabular}

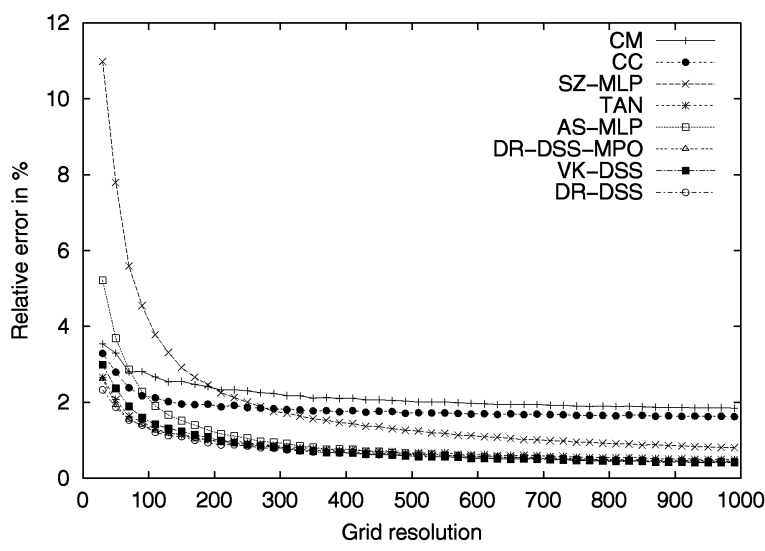

(a)

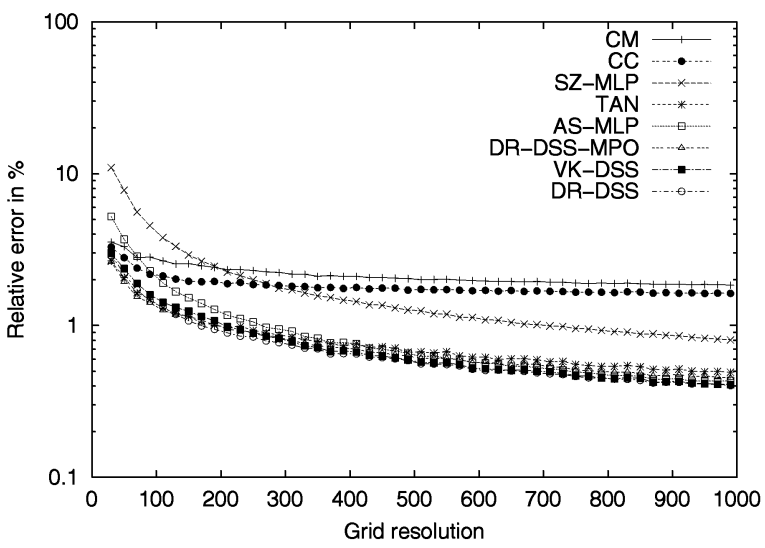

(b)

Fig. 7. Evident multigrid convergence of length estimators: (a) sliding means of relative errors, also shown in (b) a logarithmic scale.

- $3 D$ extension: May the approach be extended to length estimation of digital curves in 3D space?

Table 1 explains the situation. The convergence speed is known to be linear for DR-DSS, VK-DSS, SZ-MLP, and AS-MLP; see the cited Theorems 1, 2, 3, and 4.

We consider two measures: 1) the relative error in percent between estimated and true curve length and 2) for DSS- and MLPapproaches, also a trade off measure defined as the product of relative error times the number of generated segments (in [25], [27], it has been called the efficiency of convergence). Test data (as used in [27]) are shown in Fig. 2.

In Fig. 7, experimental convergence is evident for all methods. However, in case of methods CM and CC, we have convergence to a false value! Errors are calculated for all curves, transformed into a mean value for a given grid size, and curves are generated by sliding means (of 30 values) along different grid sizes. We show a decimal and a logarithmic scale of errors. DR-DSS-MOP slightly overestimates the length in comparison to DR-DSS.

The trade off measure is presented in Fig. 8 for polygonal approaches only. Again, values are calculated for all curves, transformed into a mean value for a given grid size, and curves are generated by sliding means (of 30 values) along different grid sizes.

As an additional test, we also rotated a square of fixed size in a grid of resolution 128. Fig. 9a shows the behavior of estimated perimeters for such a rotated square curve, for different estimation algorithms. The ideal situation would be a totally orientationindependent estimator and all methods besides CM and CC approximate this reasonably well.

Fig. $9 \mathrm{~b}$ summarizes the runtimes of polygonalization-based estimators compared to a local-metric estimator. Obviously, both local metric algorithms are the fastest. Method SZ-MLP in the implementation of [27] provides the fastest global estimator, but VK-DSS and DR-DSS are close. The AS-MLP algorithm has not yet been optimized and faster implementations might be possible. Theoretically, it is known that the TAN method allows a linear asymptotic runtime implementation [26]. However, the used program showed quadratic runtime. Further algorithmic optimization is needed.

\section{Conclusions}

The experiments show that both local-metrics approaches are not multigrid convergent (already for the test data set), but all five global

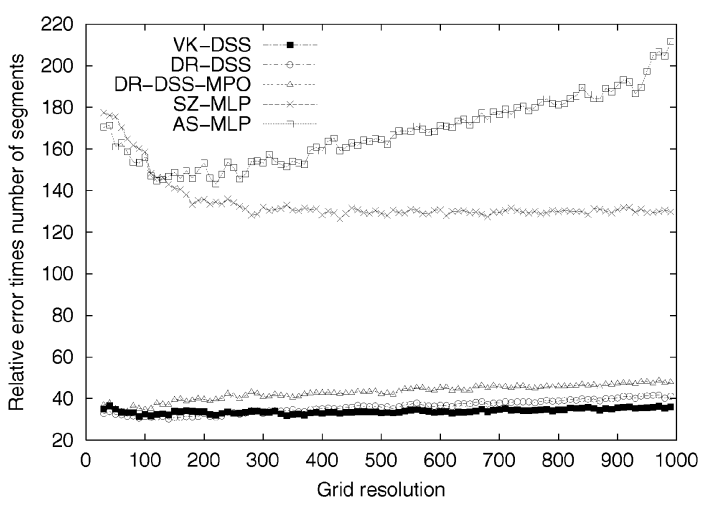

Fig. 8. Trade off value diagrams for polygonal approaches. 


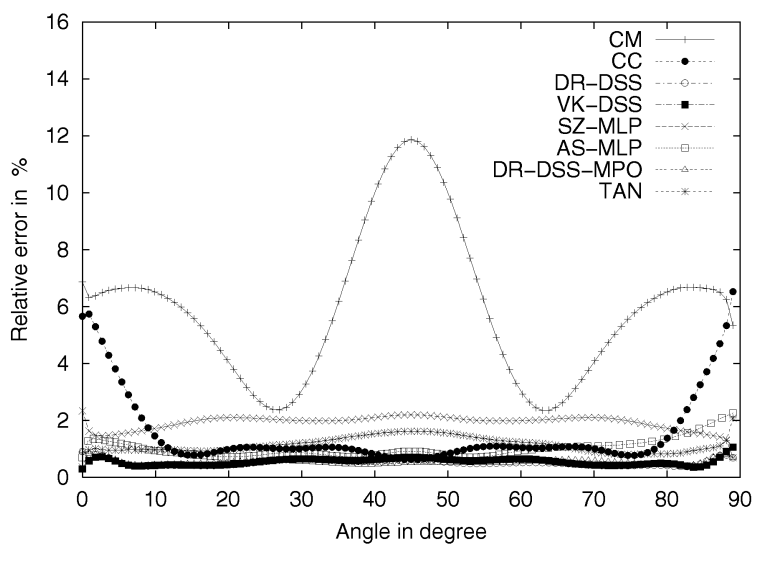

(a)

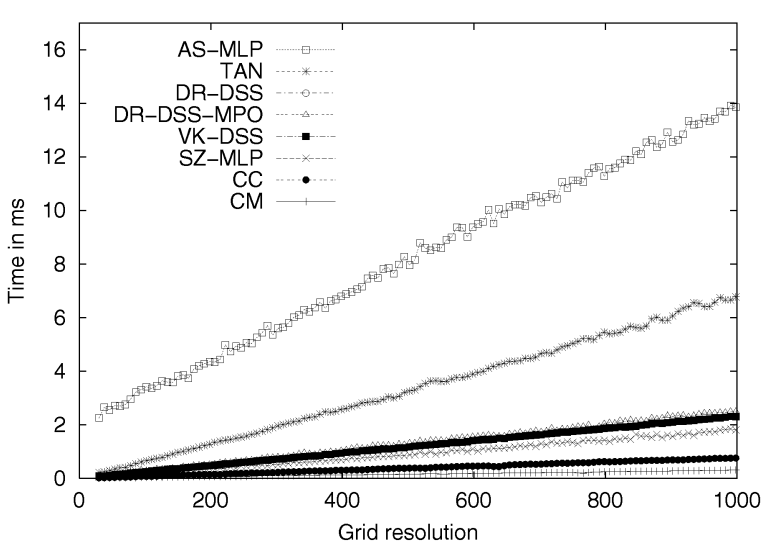

(b)

Fig. 9. (a) Relative errors for a rotating square. (b) Runtimes of polygonalization-based estimators on an Ultra 10 Sparc Workstation.

approximation methods confirm known theoretical convergence results by experimental evidence. A mathematical analysis is (still) unpublished which describes local methods that cannot achieve multigrid convergence if input data are "sufficiently complex," e.g., not just isothetic rectangles (actually there was a seminar presentation at Dagstuhl/Wadern in April 2002 by M. Tajine from Strasbourg showing that local methods cannot ensure multigrid convergence in specified situations of measurements-however, this has not yet been published). The potential failure of local methods is well-known in mathematics for 3D surface area estimates since the end of the 19th century, see, e.g., cited work of Schwarz in [34].

Interestingly, the increase in runtimes of the studied polygonal methods is only minor compared to that of local-metric algorithms. Hence, the use of a (incorrect) local-metric algorithm is also not justified by a runtime argument. The choice of a global method may depend on preferences defined by the context of an image processing software package and the authors can recommend any of the five studied global methods. Studies on test data might be useful for selecting the most efficient implementation for a given application context.

Originally, the authors also intended to use a third measure for comparison, the minimum value $r_{0}$ of grid resolution $r$ such that a method estimates $\pi$ the first time within the error interval defined by (1) (i.e., where a circular region is digitized in a grid with edge length $1 / r)$, what might be called the Archimedes-Hui constant of the algorithm implementing a method. However, due to oscillations of calculated estimations, results of an algorithm may be outside of this interval again for $r>r_{0}$ for an Archimedes-Hui constant $r_{0}$ of this algorithm and just using a sliding mean can also not be recommended because of unsecured knowledge on the general behavior of the measured error sequence. A methodically correct introduction of such an Archimedes-Hui constant remains an open problem.

\section{ACKNOWLEDGMENTS}

At the time this research was done, D. Coeurjolly was a member of the ERIC Laboratory (Université Lumière Lyon 2).

\section{REFERENCES}

[1] H. Freeman, "Techniques for the Digital Computer Analysis of ChainEncoded Arbitrary Plane Curves," Proc. Nat'l Electronics Conf., vol. 17, pp. 421-432, 1961.

[2] A. Rosenfeld, "Digital Straight Line Segments," IEEE Trans. Computers, vol. 23 , pp. 1264-1269, 1974

[3] J. Sklansky and D.F. Kibler, "A Theory of Nonuniformly Digitized Binary Pictures," IEEE Trans. Systems, Man, and Cybernetics, vol. 6, pp. 637-647, 1976.
[4] T.J. Ellis, D. Proffitt, D. Rosen, and W. Rutkowski, "Measurement of the Lengths of Digitized Curved Lines," Computer Graphics and Image Processing, vol. 10, pp. 333-347, 1979.

[5] A. Hübler, R. Klette, and K. Voss, "Determination of the Convex Hull of a Finite Set of Planar Points within Linear Time," EIK (Elektronische Informationsverarb. Kybernetik), vol. 17, pp. 121-140, 1981.

[6] E. Creutzburg, A. Hübler, and V. Wedler, “On-Line Erkennung Digitaler Geradensegmente in Linearer Zeit," Proc. GEOBILD '82, pp. 48-65, 1982.

[7] C.E. Kim, "On Cellular Straight Line Segments," Computer Graphics and Image Processing, vol. 18, pp. 369-391, 1982.

[8] L.D. Wu, "On the Chain Code of a Line," IEEE Trans. Pattern Analysis Machine Intelligence, vol. 4, pp. 347-353, 1982.

[9] A.M. Vossepoel and A.W.M. Smeulders, "Vector Code Probabilities and Metrication Error in the Representation of Straight Lines of Finite Length," Computer Graphics and Image Processing, vol. 20, pp. 347-364, 1982.

[10] B.L. van der Waerden, Geometry and Algebra in Ancient Civilizations. Berlin: Springer, 1983.

[11] T.A. Anderson and C.E. Kim, "Representation of Digital Line Segments and Their preimages," Computer Vision, Graphics, Image Processing, vol. 30, pp. 279-288, 1985.

[12] G. Borgefors, "Distance Transformations in Digital Images," Computer Vision, Graphics, and Image Processing, vol. 34, pp. 344-371, 1986.

[13] L. Dorst and A.W.M. Smeulders, "Length Estimators for Digitized Contours," Computer Vision, Graphics, and Image Processing, vol. 40, pp. 311-333, 1987.

[14] J. Koplowitz and A.M. Bruckstein, "Design of Perimeter Estimators for Digitized Planar Shapes," IEEE Trans. Pattern Analysis Machine Intelligence, vol. 11, pp. 611-622, 1989.

[15] V.A. Kovalevsky, "New Definition and Fast Recognition of Digital Straight Segments and Arcs," Proc. 10th Int'l Conf. Pattern Recognition, pp. 31-34, 1990.

[16] L. Dorst and A.W.M. Smeulders, "Discrete Straight Line Segments: Parameters, Primitives, and Properties," Contemporary Math., R. Melter, P. Bhattacharya, and A. Rosenfeld, eds., vol. 119, pp. 45-62, 1991.

[17] J.-P. Reveillès, "Géométrie Discrète, Calcul en Nombres Entiers et Algorithmique," Thèse d'état, Univ. Louis Pasteur, Strasbourg, 1991.

[18] B. Verwer, "Local Distances for Distance Transformations in Two and Three Dimensions," Pattern Recognition Letters, vol. 12, pp. 671-682, 1991.

[19] V. Kovalevsky and S. Fuchs, "Theoretical and Experimental Analysis of the Accuracy of Perimeter Estimates," Robust Computer Vision, Wichmann, Karlsruhe, pp. 218-242, 1992.

[20] I. Debled-Rennesson and J. Reveillès, "A Linear Algorithm for Segmentation of Digital Curves," Int'l J. Pattern Recognition and Artificial Intelligence, vol. 9, pp. 635-662, 1995.

[21] J. Françon, J.-M. Schramm, and M. Tajine, “Recognizing Arithmetic Straight Lines and Planes," Proc. Sixth Int'l Workshop Discrete Geometry for Computer Imagery, pp. 141-150, 1996.

[22] F. Sloboda and B. Zatko, "On One-Dimensional Grid Continua in $R^{2}$ " technical report, Inst. of Control Theory and Robotics, Bratislava, 1996.

[23] A. Vialard, "Geometrical Parameters Extraction from Discrete Paths," Discrete Geometry for Computer Imagery, pp. 24-35, 1996.

[24] A. Jonas and N. Kiryati, "Length Estimation in 3-D Using Cube Quantization," J. Math. Imaging and Vision, vol. 8, pp. 215-238, 1998.

[25] F. Sloboda, B. Zatko, and J. Stoer, “On Approximation of Planar OneDimensional Continua," Advances in Digital and Computational Geometry, R. Klette, A. Rosenfeld, and F. Sloboda, eds., pp. 113-160, 1998.

[26] F. Feschet and L. Tougne, "Optimal Time Computation of the Tangent of a Discrete Curve: Application to the Curvature," Discrete Geometry for Computer Imagery, pp. 31-40, 1999. 
[27] R. Klette, V. Kovalevsky, and B. Yip, "Length Estimation of Digital Curves," SPIE Proc. Vision Geometry VIII, pp. 117-129, July 1999. (expanded version available: Machine Graphics \& Vision, vol. 9, pp. 673-703, 2000.)

[28] T. Asano, Y. Kawamura, R. Klette, and K. Obokata, “A New Approximation Scheme for Digital Objects and Curve Length Estimations," Proc. Image and Vision Computing, pp. 26-31, 2000.

[29] R. Klette and T. Bülow, "Minimum-Length Polygons in Simple CubeCurves," Discrete Geometry for Computer Imagery, pp. 467-478, 2000.

[30] R. Klette and J. Zunic, "Multigrid Convergence of Calculated Features in Image Analysis," J. Math. Imaging Vision, vol. 13, pp. 173-191, 2000.

[31] T. Asano, Y. Kawamura, R. Klette, and K. Obokata, "Minimum-Length Polygons in Approximation Sausages," Proc. Fourth Int'l Workshop Visual Form, pp. 103-112, 2001.

[32] D. Coeurjolly, I. Debled-Rennesson, and O. Teytaud, "Segmentation and Length Estimation of 3D Discrete Curves," Proc. Digital and Image Geometry, pp. 299-317, 2001.

[33] D. Coeurjolly and O. Teytaud, "Multigrid Convergence of Discrete Differential Estimators: Discrete Tangent and Length Estimation," Research Report Laboratoire ERIC, Universite Lumiere Lyon, France, RR-0101, http:/ / eric.univ-lyon2.fr/RR/RR-0101.ps.gz, 2001.

[34] R. Klette, "Multigrid Convergence of Geometric Features," Proc. Digital and Image Geometry, pp. 318-338, 2001.

For more information on this or any other computing topic, please visit our Digital Library at http://computer.org/publications/dlib. 